Check for updates

Cite this: RSC Adv., 2018, 8, 28797

Received 21st June 2018

Accepted 4th August 2018

DOI: $10.1039 / \mathrm{c} 8 \mathrm{ra0} 3318 \mathrm{a}$

rsc.li/rsc-advances

\section{Layer charge robust delamination of organo-clays $\uparrow$}

\author{
Matthias Daab, Natalie J. Eichstaedt, Andreas Edenharter, Sabine Rosenfeldt \\ and Josef Breu (D)*
}

To date delamination of organo-clays is restricted to highly charged, vermiculite-type layered silicates (e.g. $n$-butylammonium vermiculites) while - counterintuitively - low charged, smectite-type layered silicates do not delaminate although their Coulomb interactions are much weaker. Guided by previous findings, we now identified organo-cations that allowed for extending the delamination of organo clays to charge densities in the regime of low charged smectites as well. Upon intercalation of protonated amino-sugars like $\mathrm{N}$-methyl-D-glucamine (meglumine) robust delamination of $2: 1$ layered silicates via repulsive osmotic swelling in water is achieved. This process is stable over a wide range of charge densities spanning from smectites (layer charge $x \sim 0.3$ charges per formula unit $\mathrm{Si}_{4} \mathrm{O}_{10} \mathrm{~F}_{2}$, p.f.u.) to vermiculites $(x$ $\sim 0.7$ p.f.u.). It is evidenced that a combination of first, a sufficiently large charge equivalent area (bulkiness) of meglumine with second, a significant hydrophilicity of meglumine leads to swelling above a threshold $d$-spacing of $\gtrsim 17.5 \AA$ in moist air (98\% r.h.). Hereby, electrostatic attraction is critically weakened, causing the onset of repulsive osmotic swelling which leads to utter delamination. Moreover, meglumine renders delamination tolerant to charge heterogeneities typically found in natural and synthetic clays.

\section{Introduction}

For artificial nacre materials ${ }^{1-3}$ or high-end gas-barriers, ${ }^{4-6}$ highly-filled nanocomposites of well oriented nanosheets perform best. Self-assembly into such textured films requires strict control of layer thickness. The same applies to the fabrication of heterostructures. ${ }^{7-10}$ For charged 2-dimensional (2D) materials this kind of control of delamination is feasible via osmotic swelling. ${ }^{11-13}$ This type of thermodynamically favoured disintegration of a 1D-crystalline stack of layers leads to nanosheets of structure-inherent thickness. Osmotic swelling is reported for 2D-materials of different electronic ${ }^{14-16}$ and magnetic ${ }^{17-20}$ properties like clay minerals, ${ }^{21-25}$ lepidocrocitetype titanate ${ }^{26-28}$ or layered antimony phosphates. ${ }^{29}$ During osmotic swelling the lateral extension of the nanosheets is inherited from the lateral dimension of the parent material. Thus the aspect ratio $\alpha$ (ratio of lateral dimensions to height) of the resulting nanosheets is maximized, which is particular advantageous for gas barrier applications where permeability is expected to decrease by $\alpha^{2} .{ }^{30}$ The aspect ratio can be increased by exfoliation and maximized by delamination ${ }^{31}$ and the degree

Bavarian Polymer Institute and Department of Chemistry, University of Bayreuth, D-95440 Bayreuth, Germany.E-mail: Josef.Breu@uni-bayreuth.de; Tel: +49921 55 2530

$\dagger$ Electronic supplementary information (ESI) available: PXRD of clay minerals, layer charge determination: charge density vs. chain length, meglumine-clays: CHN and EDX, volume fraction of gels (calculation), glucosamine-clays and

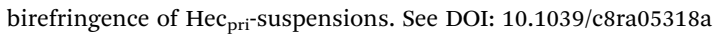

of exfoliation influences the properties of polymer nanocomposites with layered materials. . $^{6,32-37}$

Unfortunately, the viability of osmotic swelling is highly sensitive to the charge density of the layered material. For instance, for Na-hectorite $\left(\mathrm{Na}_{0.5}{ }^{\text {inter. }}\left[\mathrm{Mg}_{2.5} \mathrm{Li}_{0.5}\right]^{\text {oct. }}\left\langle\mathrm{Si}_{4}\right\rangle^{\text {tetr. }} \mathrm{O}_{10} \mathrm{~F}_{2}\right.$, $\mathrm{Na}-\mathrm{Hec}$ ) superb charge homogeneity has to be assured by a melt synthesis being followed by a lengthy high temperature annealing. ${ }^{12,38}$ Only then a clay is obtained that spontaneously and utterly delaminates upon immersion into water delivering high aspect ratio nanosheets. This filler in turn warrants superior gas barrier properties of nanocomposites. ${ }^{39}$ Even slight variations of the charge density will lead to domains with a charge above the established upper limit $(x>0.55$ per formula unit $\mathrm{Si}_{4} \mathrm{O}_{10} \mathrm{~F}_{2}$, p.f.u. ${ }^{40}$ for osmotic swelling and these domains might pin the stacks together by the strong Coulomb interactions. ${ }^{12}$ Therefore, melt-synthesized Na-hectorites that have not been homogenized by annealing do not delaminate completely. ${ }^{38,41}$

More recently and despite the even stronger Coulombattraction, we could show that more highly charged vermiculite-type clays (Na-Verm) can be utterly delaminated after ion-exchange with selected bulky and hydrophilic organocations. ${ }^{42}$ Besides the organo-cation being sufficiently hydrophilic, a threshold separation has to be achieved at which the counterion translational entropy and hydration may dominate over the Coulomb attraction. To accomplish that separation, the ratio of charge equivalent area of the interlayer cation $\left(A_{\mathrm{I}}\right.$, area that is occupied by a flat lying cation) and the charge equivalent area of the clay $\left(A_{\mathrm{C}}\right.$, area per charge as calculated by 
the charge density), $A_{\mathrm{I}} / A_{\mathrm{C}}$ should be $\gtrsim 1$, to yield a sufficient steric demand upon hydration.

Unfortunately, all organo-cations that were tested so far only delaminate highly charged vermiculites but failed to osmotically delaminate low charged, smectite-type clays like Na-Hec. Organo-cations that do the job for a broad range of charge densities would, however, be highly desirable in the light of charge heterogeneities observed for natural smectites like montmorillonite, ${ }^{\mathbf{4 3}, \mathbf{4 4}}$ for natural vermiculites, or for nonannealed synthetic Na-Hec.

Therefore, we extended and intensified our screening for a charge robust delamination agent. And in this work we introduce two ammonium-sugars that are capable to trigger osmotic swelling for charge densities ranging from smectitetype clays ( $x \sim 0.3$ p.f.u.) all the way to vermiculite-type clays $(x \sim 0.7$ p.f.u. $)$ and that moreover are tolerant to heterogeneities in charge density.

\section{Experimental}

\section{Synthesis and characterization of the clays}

A highly charged Na-fluorohectorite $\mathrm{Na}_{0.75} \mathrm{Mg}_{2.25} \mathrm{Li}_{0.75} \mathrm{Si}_{4} \mathrm{O}_{10} \mathrm{~F}_{2}$ with a nominal charge density in the range of vermiculites (Verm) and a medium charged Na-fluorohectorite $\mathrm{Na}_{0.5} \mathrm{Mg}_{2.5^{-}}$ $\mathrm{Li}_{0.5} \mathrm{Si}_{4} \mathrm{O}_{10} \mathrm{~F}_{2}$ (Hec) with a nominal charge density in the range of smectites were prepared by melt synthesis according to a published procedure followed by annealing ( 6 weeks, $1045{ }^{\circ} \mathrm{C}$ ) to improve charge homogeneity and phase purity. ${ }^{38,42}$ For the as synthesized hectorite of the nominal composition $\mathrm{Na}_{0.5} \mathrm{Mg}_{2.5^{-}}$ $\mathrm{Li}_{0.5} \mathrm{Si}_{4} \mathrm{O}_{10} \mathrm{~F}_{2}$ the annealing step after melt synthesis was left out. Instead to get rid of soluble sidephases, it was washed with $\mathrm{Na}_{4}$ EDTA (0.5 M, $50 \mathrm{~mL}$ per $1 \mathrm{~g}$ of $\mathrm{Hec}_{\text {pri }}$ ) six times at $60{ }^{\circ} \mathrm{C}$ for $48 \mathrm{~h}$ followed by washing two times with Millipore-water untill $\mathrm{pH}=6.5$ is achieved to obtain the sample $\mathrm{Hec}_{\mathrm{pri}}$.

The synthesis is carried out in gas tight molybdenum crucibles. The educts NaF (99.995\%, Alfa Aesar), LiF (>99.9\%, ChemPur), $\mathrm{MgF}_{2}$ (>99.9\%, ChemPur), $\mathrm{MgO}$ (99.95\%, Alfa Aesar) and $\mathrm{SiO}_{2}$ (Merck, fine granular quartz, purum) are mixed according to the nominal composition. The crucible was ramped to $1750{ }^{\circ} \mathrm{C}\left(15{ }^{\circ} \mathrm{C} \mathrm{min}^{-1}\right)$, held at this temperature for $70 \mathrm{~min}$, cooled to $1300{ }^{\circ} \mathrm{C}\left(55^{\circ} \mathrm{C} \mathrm{min}{ }^{-1}\right)$ and then to $1050{ }^{\circ} \mathrm{C}$ $\left(10{ }^{\circ} \mathrm{C} \min ^{-1}\right)$. Finally, it was quenched by switching of the power.

Synthesis of charge reduced clays: $5 \mathrm{~g}$ of Hec were exchanged 7 times with $400 \mathrm{~mL}$ of $2 \mathrm{M} \mathrm{MgCl}_{2}$-solution. The resulting $\mathrm{Mg}$ exchanged hectorite was washed with water till the chloridetest (with $\mathrm{AgNO}_{3}$ ) of the supernatant solution was negative. The suspension was dried at $80{ }^{\circ} \mathrm{C}$. The dried powder was heated at $250{ }^{\circ} \mathrm{C}$ for $24 \mathrm{~h}$ for charge reduction (obtained sample: LCR1). ${ }^{45} 50 \%$ of the LCR1 sample were again exchanged 7 times with $400 \mathrm{~mL}$ of $2 \mathrm{M} \mathrm{MgCl}_{2}$-solution. The resulting $\mathrm{Mg}$ exchanged hectorite was washed with water till the chloridetest of the supernatant solution was negative. The suspension was dried at $80{ }^{\circ} \mathrm{C}$. The dried powder was heated at $250{ }^{\circ} \mathrm{C}$ for $24 \mathrm{~h}$ for charge reduction (obtained sample: LCR2).
The cation exchange capacity was determined according to DIN ISO 11260 using $\mathrm{BaCl}_{2}$ and the layer charge was determined according to Lagaly. ${ }^{46,47}$

\section{Powder X-ray diffraction}

Powder X-ray diffraction (PXRD) patterns of as-synthesized clays were recorded on a STOE Stadi P powder diffractometer using $\mathrm{Cu} \mathrm{K} \alpha_{1}$ radiation. The samples were placed in a glass capillary. Prior to measurement samples were equilibrated for one week over saturated $\mathrm{K}_{2} \mathrm{CO}_{3}$-solution ( $43 \%$ relative humidity, r.h.) to obtain the one water layer hydrate. After equilibration, the capillaries were sealed. All other PXRD were measured with textured samples in Bragg-Brentano geometry on a PANalytical X'pert Pro equipped with an X'Celerator Scientific RTMS detector ( $\mathrm{Cu} \mathrm{K \alpha}$ radiation). PXRD patterns at fixed relative humidities were recorded in a temperature-humidity chamber (Anton Paar temperature humidity chamber driven by a VTI corp. RH-200 humidity generator) mounted on the PANalytical Xpert Pro. Dry samples were obtained by drying at $110^{\circ} \mathrm{C}$ under vacuum for $24 \mathrm{~h}$ and PXRDs of the hot samples were measured within 2 min each after removal from the vacuum furnace.

\section{Microprobe analysis}

The chemical composition of Hec and Verm was determined by wavelength dispersive X-ray spectroscopy on single crystals with flat lying surfaces on a carbon tape using an electron microprobe (Jeol JXA-8200, Bayerisches Geoinstitut, Bayreuth). The settings were $15 \mathrm{kV}$ acceleration voltage, $15 \mathrm{nA}$ initial beam current, a beam spot diameter of $10 \mu \mathrm{m}, 10 \mathrm{~s}$ counting time at the peak position and $5 \mathrm{~s}$ counting time at each side of the peak.

\section{Inductive-coupled plasma atomic emission spectroscopy (ICP- AES)}

The lithium and magnesium content of Hec and Verm was determined by ICP-OES. Samples of about $20 \mathrm{mg}$ of dry clay were weighed into clean Teflon flasks of $15 \mathrm{~mL}$ volume. After addition of $1.5 \mathrm{~mL} 30 \mathrm{wt} \% \mathrm{HCl}$ (Merck), $0.5 \mathrm{~mL}$ of $85 \mathrm{wt} \%$ $\mathrm{H}_{3} \mathrm{PO}_{4}$ (Merck), $0.5 \mathrm{~mL} 65$ wt\% $\mathrm{HNO}_{3}$ (Merck) and $1 \mathrm{~mL}$ of 48 wt\% HBF4 (Merck) the sample was digested in a MLS 1200 Mega microwave digestion apparatus for $6.5 \mathrm{~min}$ and heated at 600W (MLS GmbH, Mikrowellen-Labor-Systeme, Leutkirch, Germany). The closed sample container was cooled to room temperature and the clear solution was diluted to $100 \mathrm{~mL}$ in a volumetric flask and analyzed on a Vista-PRO radial spectrometer.

\section{Cation-exchange with meglumine}

$200 \mathrm{mg}$ of the dry, pristine clays were suspended in $50 \mathrm{~mL}$ of a $1 \mathrm{M}$ solution of $\mathrm{N}$-methyl-D-glucamine (meglumine, in its protonated form, $\mathrm{pH}=7,>125$-fold excess of the CEC, delamination is prevented by the high ionic strength). The procedure was repeated 5 times. Finally, the obtained organoclays were washed free of chloride-ions $\left(\mathrm{AgNO}_{3}\right.$-test) with Millipore-water. Gels were concentrated by centrifugation 
between the washing cycles. The organo-clays were finally washed one times with acetone and dried at $60{ }^{\circ} \mathrm{C}$.

\section{CHN-analysis}

A PerkinElmer $2400 \mathrm{CHN}$ equipped with a combustion tube filled with tungsten(vi)-oxide-granules was used at a combustion temperature of $1050{ }^{\circ} \mathrm{C}$. Samples were dried at $110{ }^{\circ} \mathrm{C}$ and vacuum for $24 \mathrm{~h}$ and stored in a glovebox prior to measurement.

\section{Scanning electron microscopy and energy dispersive X-ray spectroscopy (SEM-EDX)}

SEM-EDX was measured on a Zeiss 1530 with a EDX INCA 400 unit (Oxford). Samples were prepared on a carbon tape and sputtered with $10 \mathrm{~nm}$ carbon.

\section{Small angle X-ray scattering (SAXS)}

SAXS data were measured using a "Double Ganesha AIR" system (SAXSLAB, Denmark). The X-ray source of this laboratory-based system is a rotating anode (copper, MicroMax 007HF, Rigaku Corporation, Japan) providing a micro-focused beam. The data are recorded by a position sensitive detector (PILATUS 300K, Dectris). Samples were prepared by adding a defined amount of millipore-water to the dry organo-clay. After equilibration for one week SAXS-patterns were recorded in $1 \mathrm{~mm}$ glass capillaries.

\section{Atomic force microscopy (AFM)}

AFM was done in tapping mode using a Dimension 3100 NanoScope IV-unit equipped with OTESPA-R3 (Bruker) silicon tips. Samples were prepared by dropping a very dilute aqueous dispersion ( $5 \mathrm{mg} \mathrm{L}^{-1}$ ) in millipore water onto silicon wafers.

\section{Results and discussion}

\section{Synthesis and characterization of the clays}

Clays with layer charges in the range $0.75>x>0.3$ p.f.u. have been synthesized. Highly charged Verm $\mathrm{Na}_{0.75}{ }^{\text {inter. }}\left[\mathrm{Mg}_{2.25}\right.$ $\left.\mathrm{Li}_{0.25}\right]^{\text {oct. }}\left\langle\mathrm{Si}_{4}\right\rangle^{\text {tetr. }} \mathrm{O}_{10} \mathrm{~F}_{2}(x=0.75$ p.f.u. nominally $)$ and medium charged Hec $\mathrm{Na}_{0.5}{ }^{\text {inter. }}\left[\mathrm{Mg}_{2.5} \mathrm{Li}_{0.5}\right]^{\text {oct. }}\left\langle\mathrm{Si}_{4}\right\rangle^{\text {tetr. }} \mathrm{O}_{10} \mathrm{~F}_{2}(x=0.5$ p.f.u. nominally) are directly accessible via melt synthesis. Both were homogenized post-synthesis by long term-annealing in a gas tight crucible to prevent loss of volatile fluorides at elevated temperatures. ${ }^{38,42}$ Their analysis is recapitulated briefly. As reported before, PXRDs (Fig. S1 $\dagger$ ) indicate a crystalline hydrate for both clays with one layer of water molecules in the interlayer space at $43 \%$ relative humidity (r.h.) $\left(d_{001}=12.5 \AA\right) .{ }^{48}$ The patterns can be indexed in $C 2 / m$ (no. 12) and the diffractograms can be completely indexed; least squares refined lattice parameters for Verm are $a=5.26(1) \AA, b=9.07(2) \AA, c=12.48(4)$ $\AA$ А, $\beta=95.22(4)^{\circ}$ and for Hec $a=5.20(1) \AA, b=9.10(1) \AA, c=$ 12.48(4) $\AA$, $\beta=95.44(4)^{\circ}$. The chemical composition was determined by microprobe analysis. Microprobe analysis is insensitive to $\mathrm{Li}$. Therefore, the $\mathrm{Mg} / \mathrm{Li}$-ratio was derived from ICP-OES analysis by assuming a complete occupancy of the tetrahedral positions $(\mathrm{Si}=4)$ and by normalizing stoichiometric coefficients of octahedral and interlayer cations to match the 22 negative charges of the anionic sublattice $\left(\mathrm{O}_{10} \mathrm{~F}_{2}\right)$. This way compositions of $\mathrm{Na}_{0.71(4)} \mathrm{Mg}_{2.28(4)} \mathrm{Li}_{0.72(4)} \mathrm{Si}_{4} \mathrm{O}_{10} \mathrm{~F}_{2}$ and of $\mathrm{Na}_{0.52(2)} \mathrm{Mg}_{2.51(4)} \mathrm{Li}_{0.46(2)} \mathrm{Si}_{4} \mathrm{O}_{10} \mathrm{~F}_{2}$ were obtained for annealed Verm and annealed $\mathrm{Hec}$, respectively.

Lower charged clays that are not directly accessible via melt synthesis, ${ }^{45}$ were obtained by layer charge reduction (LCR) of annealed Hec by an anti-Hofmann-Klemen-effect: In doing so, $\mathrm{Hec}$ is first ion exchanged with $\mathrm{Mg}^{2+}$ followed by annealing at $250{ }^{\circ} \mathrm{C}$ whereupon octahedral $\mathrm{Li}^{+}$and interlayer $\mathrm{Mg}^{2+}$ exchange sites. With increasing incorporation of $\mathrm{Mg}^{2+}$ into the octahedral layer, concomitantly the permanent negative charge of the silicate layers is reduced. ${ }^{45}$ These samples were named LCR1 and LCR2 corresponding to one and two cycles of charge reduction.

Moreover, a pristine Na-hectorite that has not been homogenized by annealing was included as a reference ( $\mathrm{Hec}_{\mathrm{pri}}$ ) (Fig. S2 $\dagger$ ). The composition was determined as $\mathrm{Na}_{0.55(1)}{ }^{\text {inter. }}\left[\mathrm{Mg}_{2.48(2)} \mathrm{Li}_{0.49(2)}\right]^{\text {oct. }}\left\langle\mathrm{Si}_{4}\right\rangle^{\text {tetr. }} \mathrm{O}_{10} \mathrm{~F}_{2}$.

The cation exchange capacities (CEC) were determined by the $\mathrm{BaCl}_{2}$-method were found to be $185 \mathrm{meq} / 100 \mathrm{~g}$ for Verm, 129 $\mathrm{meq} / 100 \mathrm{~g}$ for $\mathrm{Hec}, 103 \mathrm{meq} / 100 \mathrm{~g}$ for LCR1, $75 \mathrm{meq} / 100 \mathrm{mg}$ for LCR2 and $116 \mathrm{meq} / 100 \mathrm{~g}$ for $\mathrm{Hec}_{\mathrm{pri}}$.

Owing to side phases without cation exchange capability, the experimentally determined CEC of $\mathrm{Hec}_{\text {pri }}(116 \mathrm{meq} / 100 \mathrm{~g})$ is significantly lower than of the annealed Hec (129 meq/100g).

The layer charge density was determined experimentally by the method of Lagaly ${ }^{\mathbf{4 6 , 4 7}}$ where interlayer ions are exchanged with $n$-alkylammonium $\left(\mathrm{C}_{n} \mathrm{H}_{2 n+1} \mathrm{NH}_{3}{ }^{+}\right.$, Fig. 1). For these organocations the equivalent area per charge is known for a dense packing of either mono- $(d=13.3 \AA$, dotted line $)$ or bilayers $(d=$ 17.6 $\AA$, dashed line). The onset of the transition of mono- to bilayer- or from bilayer to pseudo-trilayer arrangement with increasing chain length was converted into upper limits of charge densities (Table S1†).

For high charged Verm $n=9$ represents the longest alkylammonium chain that is still capable to balance the charge density in a bilayer $(d=17.6 \AA)$. Slightly increasing the equivalent area to $n=10$ some pseudo-trilayers have to be mixed in to warrant charge balance as evidenced by a shift of the $d$-spacing (Fig. $1 \mathrm{~A}$ ) which indicates $0.67 \leq x \leq 0.73$ p.f.u.

In a similar way, for the lower charged clays, the charge densities were derived from the transition of mono- to bilayers. with limiting chain length of $n=5$ (Hec, Fig. 1B), $n=8$ (LCR1, Fig. 1C) and $n=11$ (LCR2, Fig. 1D) corresponding to $0.50 \leq x \leq$ 0.56 p.f.u., $0.37 \leq x \leq 0.40$ p.f.u. and $0.29 \leq x \leq 0.31$ p.f.u. for Hec, LCR1 and LCR2, respectively (Table S1 $\dagger$ ). Interestingly, for LCR2 with $n=12$ two maxima are observed indicating a certain degree of charge heterogeneity. Apparently, the charge reduction process does not happen in a perfectly homogeneous way. This fact has previously been documented by a broadening of micropore size distributions of microporous organically pillared silicates derived from charge reduced hectorites. ${ }^{\mathbf{4 9}}$

Similarly to LCR2, for $\mathrm{Hec}_{\text {pri }}(0.50 \leq x \leq 0.56$ p.f.u., Fig. 1E) charge heterogeneity is reflected by a non-uniform increase in $d$ spacing at longer chain length $(n=10)$ in comparison to $\mathrm{Na}-\mathrm{Hec}$ (compare Fig. 1E and B). In particular, with $n=10$ for $\mathrm{Hec}_{\mathrm{pri}} d$ - 


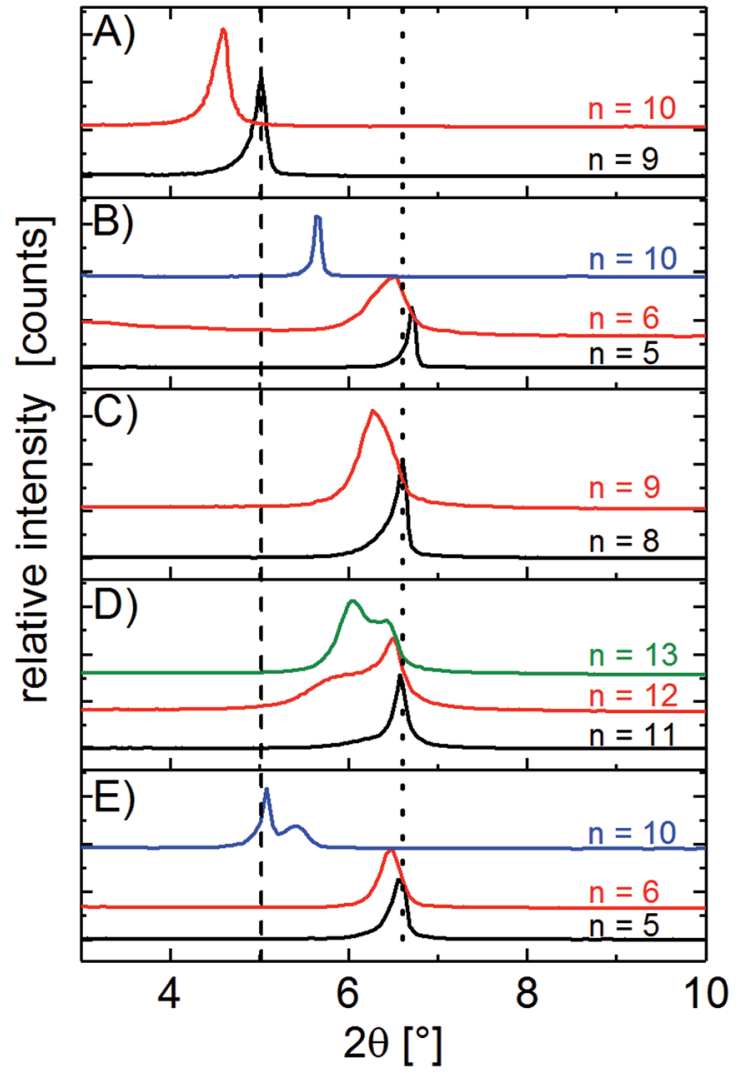

Fig. 1 Layer charge determination according to Lagaly. Interlayer ions are exchanged with $n$-alkylammonium ions of different chain length $\left(\mathrm{C}_{n} \mathrm{H}_{2 n+1} \mathrm{NH}_{3}{ }^{+}\right)$for which the equivalent area per charge is known for a dense packing of either mono- $(d=13.3 \AA$, dotted line) or bilayers ( $d$ $=17.6 \AA$, dashed line). (A) Verm, (B) Hec, (C) LCR1, (D) LCR2, (E) Hec pri.

spacings were observed that are significantly shifted to higher values as compared to $\mathrm{Hec}$. This indicates that $\mathrm{Hec}_{\mathrm{pri}}$ not only is more heterogeneous but also at least parts of the sample have a higher layer charge as compared to Hec. Please note, that this two phase behaviour of the layer charge density probably is inherent to the melt synthesis conditions and is therefore an intrinsic problem that needs to be solved by a layer charge robust delamination process: pseudo-binary alkaline and earth alkaline silicate system show pronounced immiscibility regions $s^{50}$ and the critical temperature might even increase upon addition of fluoride. ${ }^{41,51}$

\section{Swelling behaviour of organo-clays}

All clays were ion-exchanged with a hydrophilic but bulky ammonium-sugar (protonated $\mathrm{N}$-methyl-D-glucamine, referred to as meglumine). The expected meglumine contents (in $\mathrm{mmol} /$ $100 \mathrm{~g}$ ) as calculated from the Ba-CEC (Table S2 $\dagger$ ) are in fair agreement with meglumine contents as determined by $\mathrm{CHN}$ analysis. Verm, Hec, LCR1, LCR2 and $\mathrm{Hec}_{\text {pri }}$ contained 138, 104, 90, 69 and $89 \mathrm{mmol}$ meglumine/100 g, (expected: 140, 105, 87, 66 and $97 \mathrm{mmol} / 100 \mathrm{~g}$, respectively). The Na-content of NaVerm, Na-Hec and $\mathrm{Na}-\mathrm{Hec}_{\text {pri }}$ was below the detection limit of EDX (Fig. S3†). Which indicates that ion-exchange is complete within analytical accuracy.

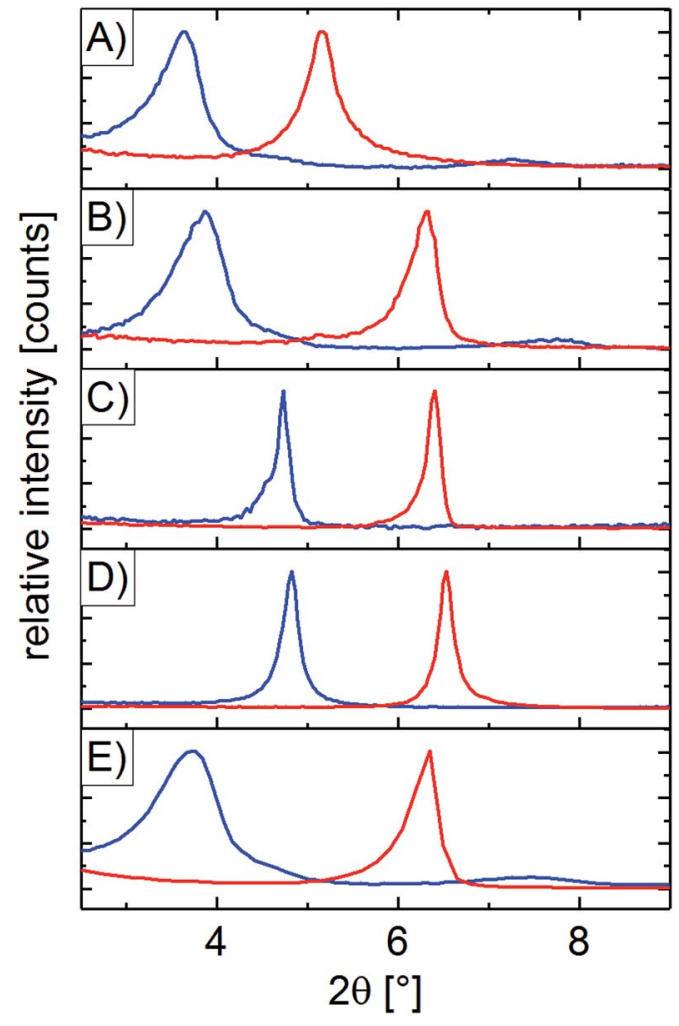

Fig. 2 PXRD of meglumine-clays (dry, red trace and at $98 \%$ r.h., blue trace). (A) Verm, (B) Hec, (C) LCR1, (D) LCR2, (E) Hec pri.

The swelling of meglumine-clays is significant (Fig. 2) even in humid air (98\% r.h.). The $d$-spacings increase from $16.8 \AA$ to 24.4 $\AA$ (Verm), 14.1 to $22.8 \AA$ (Hec), 13.7 to $18.7 \AA$ (LCR1), 13.4 to 18.2 $\AA$ (LCR2) and from 14.0 ̊ to $23.6 \AA$ ( $\mathrm{Hec}_{\text {pri }}$ ). Furthermore, the significant increase in $d$-spacing upon swelling of $\Delta d=7.6 \AA$ (Verm), $8.7 \AA$ (Hec), $5.0 \AA$ (LCR1), $4.8 \AA$ (LCR2) and $9.6 \AA$ ( $\mathrm{Hec}_{\text {pri) }}$ may be attributed to the steric pressure of meglumine upon hydration as explained in the following:

With $\mathrm{Jmol}^{52}$ a charge equivalent area of the interlayer cation meglumine as high as $A_{\mathrm{I}}=77 \AA^{2}$ can be estimated for a flat lying meglumine cation (van-der-Waals extensions $13.5 \AA \times 5.7 \AA$ ). Consequently, even in case of low charged LCR $2(x \sim 0.3$ p.f.u., corresponding to a charge equivalent area of the clay of $A_{\mathrm{C}}=79$ $\AA^{2} /$ charge) charge balance requires a densely packed monolayer of meglumine $\left(A_{\mathrm{I}} / A_{\mathrm{C}} \sim 1\right)$.

In the dry state no significant voids are available that could accommodate water molecules. Therefore, upon hydration of the hydrophilic interlayer cation at elevated r.h., expansion of the $d$-spacing is compulsory. The maximum $d$-spacings of meglumine-clays after water uptake from humid air are $>17.5 \AA$ for all clays of varying charge density (Fig. 2). This $d$-spacing was suggested to be required for rendering the interaction of adjacent silicate layers repulsive once immersed into liquid water due to contributions of repulsive counter ion translational entropy at large layer separations and critical weakening electrostatic attraction. ${ }^{42}$

Meglumine therefore is the first organo-cation that fulfils all necessary requirements postulated for allowing repulsive 

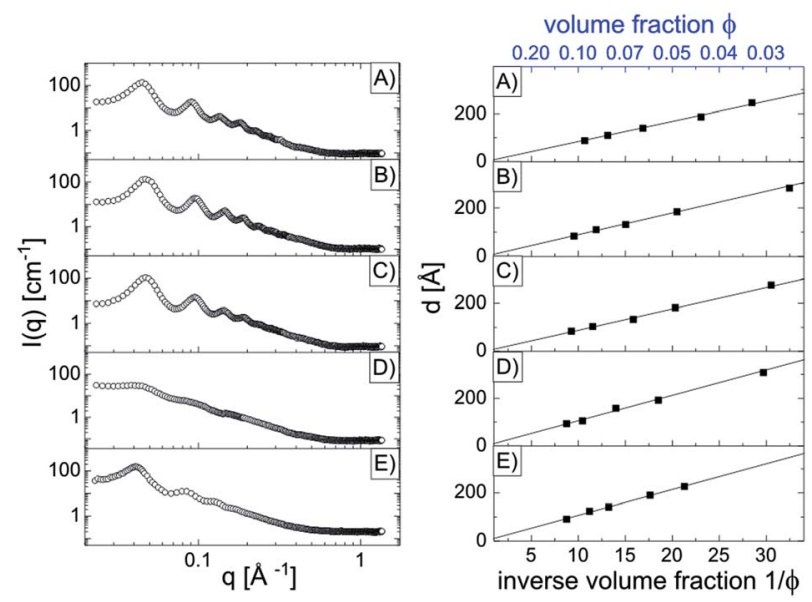

Fig. 3 Left: SAXS-pattern of Verm (A, 5.9 vol\%), Hec (B, 6.7 vol\%), LCR1 (C, 6.3 vol\%), LCR2 (D, 7.1 vol\%) and $\mathrm{Hec}_{\mathrm{pri}}(\mathrm{E}, 7.6 \mathrm{vol} \%)$. Right: The slope of $d v s .1 / \phi$ is $8.4 \AA$ (Verm, A), $8.8 \AA$ (Hec, B), $8.9 \AA$ (LCR1, C), $10.5 \AA$ (LCR2, D) and $10.7 \AA$ ( $\mathrm{Hec}_{\text {pri, }}$ E).

osmotic delamination of organo-clays even for low charged ones. ${ }^{42}$ Most importantly, the requirements are met for a broad range of charge densities ranging from low-charged smectites all the way to highly charged vermiculite-type charges.

And indeed, repulsive osmotic swelling could be confirmed for all clays, also for low charged, smectite-type clays where other organo-cations investigated previously failed: when immersing meglumine-clays into water homogeneous gels consisting of one dimensional liquid crystals are obtained for all layer charges (Fig. 3, left). Their structure is explained by SAXS-data as follows:

Due to the repulsive nature of adjacent like-charged silicate layers, these layers are separated to their maximum distance which is defined by the clay in water-volume fraction $(\varphi$, Fig. 3 , right). Even at quite low clay content, the platelets cannot freely rotate because of the typical lateral dimension $(>10 \mu \mathrm{m})$ are orders of magnitude larger than the separation of adjacent clay platelets. This separation can therefore be observed in the SAXS patterns as a series of 001 reflections with $d$-spacings of $140 \AA$ (Verm, Fig. 3A, left), $132 \AA$ (Hec, Fig. 3B, left), $133 \AA$ (LCR1, Fig. 3C, left), $158 \AA$ (LCR2, Fig. 3D, left) and $141 \AA$ ( $\mathrm{Hec}_{\mathrm{pri}}$, Fig. 3E, left) at concentrations of $5.9 \mathrm{vol} \%$ (Verm), $6.7 \mathrm{vol} \%$ (Hec), 6.3 vol\% (LCR1), 7.1 vol\% (LCR2) and 7.6 vol\% $\left(\mathrm{Hec}_{\mathrm{pri}}\right)$. (For details of conversion of wt\% into vol\% see ESI $\dagger$ ).

At clay contents $>3 \mathrm{vol} \%$, the $d$-spacing is indirect proportional to $\varphi .^{11,12}$ Taking into account the thickness of the silicate layer $(t=9.6 \AA)$ the separation of adjacent layers is given by $d=$ $t / f$. For the given vol\%, d-values of $163 \AA$ (Verm), $143 \AA$ (Hec), 152 $\AA$ (LCR1), $135 \AA$ (LCR2) and $127 \AA$ ( $\mathrm{Hec}_{\mathrm{pri}}$ ) are expected, respectively. These values are in fair agreement with observed $d$ spacings for all clays. This is a first indication that the gels consist of domains of lamellar liquid crystals with a homogeneous separation of adjacent silicate layers.

This is furthermore corroborated by studying the increase of $d$-spacings of adjacent silicate layers upon increasing the water content (Fig. 3, right). A linear slope was found as expected according to $d=t / f$. The experimentally determined slopes are

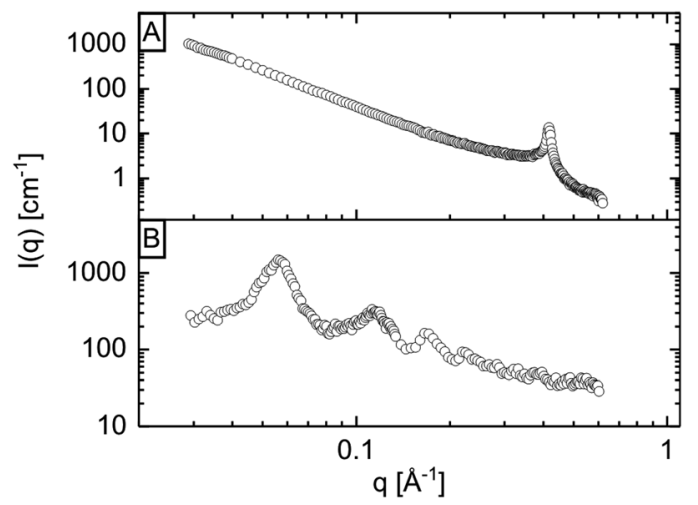

Fig. 4 SAXS-patterns. (A) For $\mathrm{Na}-\mathrm{Hec}_{\text {pri }}$ only a crystalline hydrate (two water layer hydrate, $15 \AA$ ) is found, while (B) $\mathrm{Na}$-Hec swells osmotically in water ( $d=112 \AA, 8.4$ vol\%, expected $d$-spacing: $114 \AA$ ).

$8.4 \AA$ (Verm, Fig. 3A, right), $8.8 \AA$ (Hec, Fig. 3B, right), $8.9 \AA$ (LCR1, Fig. 3C, right), $10.5 \AA$ (LCR2, Fig. 3D, right) and $10.7 \AA$ $\left(\mathrm{Hec}_{\text {pri }}\right.$, Fig. 3E, right) which is in good agreement with the expected value of $9.6 \AA$. Utter osmotic swelling to homogeneous, mono-phase gels is therefore concluded for all meglumine-clays with various charge densities.

$\mathrm{Hec}_{\text {pri }}$ represents a perfect test case for demonstrating the robustness and tolerance towards charge density variations for repulsive osmotic delamination with meglumine. Contrary to Na-Hec $(d=112 \AA$, 8.4 vol\%, expected $d$-spacing: $114 \AA$ ), Na$\mathrm{Hec}_{\text {pri }}$ does not delaminate completely when immersed into liquid water although the charge density of both Na-clays is comparable (Fig. 4). Moreover, swelling of $\mathrm{Na}-\mathrm{Hec}_{\text {pri }}$ is limited to a crystalline hydrate (two water layer hydrate, $15 \AA^{, 53-56}$ Fig. 4A), although a minor fraction $(<5 \mathrm{wt} \%$ of total solid content) probably delaminates upon immersion in water, as indicated by the formation of a birefringent suspension between crossed polarizers (Fig. S4†). Anyhow, while most of $\mathrm{Hec}_{\text {pri }}$ forms a non-birefringent sediment upon immersion in water. Its heterogeneity is emphasized by the already mentioned partial delamination. However, it should be highlighted, that meglumine exchanged $\mathrm{Hec}_{\text {pri }}$ delaminates completely (Fig. 3E) in contrast to $\mathrm{Na}-\mathrm{Hec}_{\mathrm{pri}}$ indicating its robustness against charge heterogeneities. Quite pleasing, this spares us the lengthy annealing procedure which so far was required to "activate" Na$\mathrm{Hec}_{\text {pri }}$ for osmotic swelling. ${ }^{38}$

All diluted aqueous suspensions of meglumine-clays show birefringence as expected for a lyotropic texture between crossed-polarizers at $\sim 0.25 \mathrm{vol} \%$ due to the formation of a nematic sol (Fig. 5).

Upon further dilution $\left(\sim 10^{-4}\right.$ vol\%) delamination into single platelets is evidenced by casting the then isotropic dispersions on a silicon wafer. With AFM only platelets with a height of $\sim 1 \mathrm{~nm}$ were found in good agreement with the thickness of one silicate layer $(9.6 \AA)$.

Meglumine is not unique. Other bulky and hydrophilic ammonium-sugars like glucosamine $\left(\sim 72 \AA^{2}, A_{\mathrm{I}} / A_{\mathrm{C}}=0.9\right.$ for LCR2) also have the potential of delaminating clays with broadly varying layer charge densities (Fig. S5 $\dagger$ ). 

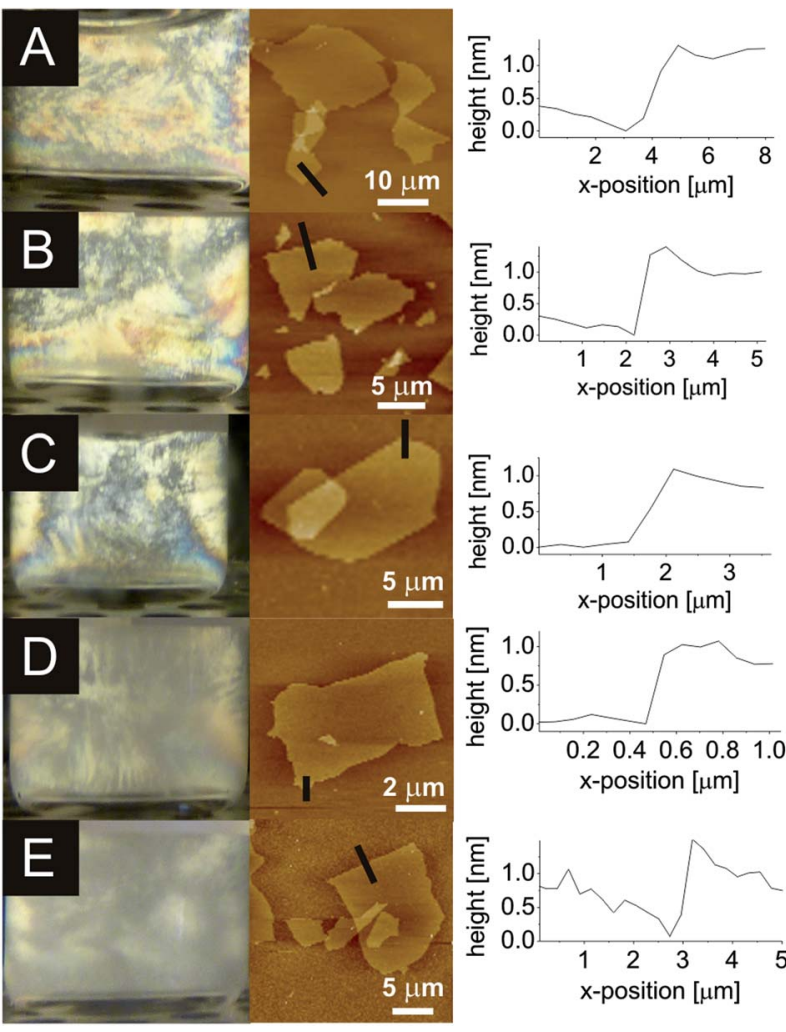

Fig. 5 Upon dilution to $\sim 0.25$ vol\% the aqueous meglumine-clay suspensions show birefringence between crossed polarizers due to the formation of a nematic sol (left), in case isotropic dispersions of $10^{-4}$ vol\% are dropped on a Si-wafer and dried, platelets are found by AFM (middle) with the height of a single-clay layer (right). (A) Verm, (B) Hec, (C) LCR1, (D) LCR2 and (E) Hec pri.

\section{Conclusions}

We have previously proposed that organo-cations that are capable of osmotic swelling in a given clay mineral are characterized by two features: they should be bulky $\left(A_{\mathrm{I}} / A_{\mathrm{C}} \gtrsim 1\right)$ and sufficiently hygroscopic. The hygroscopicity is expressed by significant swelling when going from $0 \%$ to $98 \%$ r.h. $(\Delta d \gtrsim 3.5$ $\AA)$. If then at $98 \%$ r.h. a basal threshold separation $(d \geq 17.5 \AA)$ of the corresponding organo-clay is accomplished, the gain in counter-ion translational entropy contributes to repulsive delamination upon immersion in water.

Based on the above empirical recipe, we extended our screening to ammonium-sugars. These are considerably bulkier than for instance TRIS (Tris(hydroxymethyl)ammoniummethane, $A_{\mathrm{I}}=37 \AA^{2}$ ) used before for vermiculites. This way we ensure $A_{\mathrm{I}} / A_{\mathrm{C}} \gtrsim 1$ is met. At the same time their hydrophilicity is high due to a large number of $\mathrm{OH}$-groups. Therefore, we were able to extend osmotic swelling of organo-clays into the regime of low-charged organo-smectites, which was so far only feasible for highly charged ones.

Moreover, the approach proves tolerance to charge heterogeneities. This is important from a practical point of view since natural clays like montmorillonite suffer from pronounced charge density heterogeneity. Therefore, delamination of clays by meglumine is robust in respect to a broad range of charge densities.

The results furthermore suggest, that repulsive osmotic delamination may be applied generally to charged two dimensional materials irrespective of their charge density.

\section{Conflicts of interest}

There are no conflicts to declare.

\section{Acknowledgements}

This work was supported by the Deutsche Forschungsgemeinschaft (SFB 840) and by BYK Chemie GmbH. M.D. thanks the Fonds der chemischen Industrie for a fellowship. Bayerisches Geoinstitut is acknowledged for microprobe analysis and BayCEER for ICP-OES measurements.

\section{Notes and references}

1 Z. Tang, N. A. Kotov, S. Magonov and B. Ozturk, Nat. Mater., 2003, 2, 413-418.

2 J. Wang, Q. Cheng, L. Lin and L. Jiang, ACS Nano, 2014, 8, 2739-2745.

3 H.-B. Yao, Z.-H. Tan, H.-Y. Fang and S.-H. Yu, Angew. Chem., Int. Ed., 2010, 49, 10127-10131.

4 W. S. Jang, I. Rawson and J. C. Grunlan, Thin Solid Films, 2008, 516, 4819-4825.

5 M. A. Priolo, D. Gamboa and J. C. Grunlan, ACS Appl. Mater. Interfaces, 2009, 2, 312-320.

6 E. S. Tsurko, P. Feicht, F. Nehm, K. Ament, S. Rosenfeldt, I. Pietsch, K. Roschmann, H. Kalo and J. Breu, Macromolecules, 2017, 50, 4344-4350.

7 K. S. Novoselov, D. Jiang, F. Schedin, T. J. Booth, V. V. Khotkevich, S. V. Morozov and A. K. Geim, Proc. Natl. Acad. Sci. U. S. A., 2005, 102, 10451-10453.

8 A. K. Geim and I. V. Grigorieva, Nature, 2013, 499, 419-425.

9 D. Jariwala, T. J. Marks and M. C. Hersam, Nat. Mater., 2017, 16, 170-181.

10 B. V. Lotsch, Annu. Rev. Mater. Res., 2015, 45, 85-109.

11 L. J. Michot, I. Bihannic, S. Maddi, S. S. Funari, C. Baravian, P. Levitz and P. Davidson, Proc. Natl. Acad. Sci. U. S. A., 2006, 103, 16101-16104.

12 S. Rosenfeldt, M. Stöter, M. Schlenk, T. Martin, R. Q. Albuquerque, S. Förster and J. Breu, Langmuir, 2016, 32, 10582-10588.

13 R. J. M. Pellenq, J. M. Caillol and A. Delville, J. Phys. Chem. B, 1997, 101, 8584-8594.

14 N. Sakai, Y. Ebina, K. Takada and T. Sasaki, J. Am. Chem. Soc., 2004, 126, 5851-5858.

15 T. Sasaki and M. Watanabe, J. Phys. Chem. B, 1997, 101, 10159-10161.

16 S. S. Kim, T. V. Khai, V. Kulish, Y.-H. Kim, H. G. Na, A. Katoch, M. Osada, P. Wu and H. W. Kim, Chem. Mater., 2015, 27, 4222-4228. 
17 H. Hemmen, N. I. Ringdal, E. N. De Azevedo, M. Engelsberg, E. L. Hansen, Y. Méheust, J. O. Fossum and K. D. Knudsen, Langmuir, 2009, 25, 12507-12515.

18 E. Paineau, I. Dozov, I. Bihannic, C. Baravian, M.-E. M. Krapf, A.-M. Philippe, S. Rouzière, L. J. Michot and P. Davidson, ACS Appl. Mater. Interfaces, 2012, 4, 4296-4301.

19 X. Dong, M. Osada, H. Ueda, Y. Ebina, Y. Kotani, K. Ono, S. Ueda, K. Kobayashi, K. Takada and T. Sasaki, Chem. Mater., 2009, 21, 4366-4373.

20 M. Osada, Y. Ebina, K. Fukuda, K. Ono, K. Takada, K. Yamaura, E. Takayama-Muromachi and T. Sasaki, Phys. Rev. B, 2006, 73, 153301.

21 L. Pauling, Proc. Natl. Acad. Sci. U. S. A., 1930, 16, 123-129.

22 E. Paineau, I. Bihannic, C. Baravian, A. M. Philippe, P. Davidson, P. Levitz, S. S. Funari, C. Rochas and L. J. Michot, Langmuir, 2011, 27, 5562-5573.

23 G. Lagaly and S. Ziesmer, Adv. Colloid Interface Sci., 2003, 100-102, 105-128.

24 N. Miyamoto, H. Iijima, H. Ohkubo and Y. Yamauchi, Chem. Commun., 2010, 46, 4166-4168.

25 T. Nakato and N. Miyamoto, Materials, 2009, 2, 1734.

26 F. Geng, R. Ma, Y. Ebina, Y. Yamauchi, N. Miyamoto and T. Sasaki, J. Am. Chem. Soc., 2014, 136, 5491-5500.

27 T. Maluangnont, K. Matsuba, F. Geng, R. Ma, Y. Yamauchi and T. Sasaki, Chem. Mater., 2013, 25, 3137-3146.

28 T. Sasaki and M. Watanabe, J. Am. Chem. Soc., 1998, 120, 4682-4689.

29 J. C. Gabriel, F. Camerel, B. J. Lemaire, H. Desvaux, P. Davidson and P. Batail, Nature, 2001, 413, 504-508.

30 E. L. Cussler, S. E. Hughes, W. J. Ward III and R. Aris, J. Membr. Sci., 1988, 38, 161-174.

31 J. E. F. C. Gardolinski and G. Lagaly, Clay Miner., 2005, 40, 547.

32 B. Fischer, M. Ziadeh, A. Pfaff, J. Breu and V. Altstädt, Polymer, 2012, 53, 3230-3237.

33 M. Ziadeh, B. Chwalka, H. Kalo, M. R. Schütz and J. Breu, Clay Miner., 2012, 47, 341-351.

34 H. Moustaf, A. M. Youssef and M. T. Nour, $R S C$ Adv., 2016, 6, 36467-36474.

35 A. M. Youssef, H. A. Moustafa, A. B. Barhoum, A. A. A. Hakim and A. Dufresne, ChemistrySelect, 2017, 2, 8553-8566.

36 A. M. Youssef, T. Bujdosó, V. Hornok, S. Papp, B. Kiss, A. E. A. Hakim and I. Dékány, Appl. Clay Sci., 2013, 77-78, $46-51$.
37 A. M. Youssef, F. M. Malhat, A. A. A. Hakim and I. Dékány, Arabian J. Chem., 2017, 10, 631-642.

38 M. Stöter, D. A. Kunz, M. Schmidt, D. Hirsemann, H. Kalo, B. Putz, J. Senker and J. Breu, Langmuir, 2013, 29, 1280-1285.

39 D. A. Kunz, J. Schmid, P. Feicht, J. Erath, A. Fery and J. Breu, ACS Nano, 2013, 7, 4275-4280.

40 K. Jasmund and G. Lagaly, Tonminerale und Tone: Struktur, Eigenschaften, Anwendungen und Einsatz in Industrie und Umwelt, Steinkopff, Darmstadt, 1993, p. 110.

41 J. Breu, W. Seidl, A. J. Stoll, K. G. Lange and T. U. Probst, Chem. Mater., 2001, 13, 4213-4220.

42 M. Daab, N. J. Eichstaedt, C. Habel, S. Rosenfeldt, H. Kalo, H. Schießling, S. Förster and J. Breu, Langmuir, 2018, 34, 8215-8222.

43 G. E. Christidis, A. E. Blum and D. D. Eberl, Appl. Clay Sci., 2006, 34, 125-138.

44 G. E. Christidis and D. D. Eberl, Clays Clay Miner., 2003, 51, 644-655.

45 M. M. Herling, H. Kalo, S. Seibt, R. Schobert and J. Breu, Langmuir, 2012, 28, 14713-14719.

46 A. R. Mermut and G. Lagaly, Clays Clay Miner., 2001, 49, 393397.

47 G. Lagaly, Clays Clay Miner., 1982, 30, 215-222.

48 H. Kalo, W. Milius and J. Breu, RSC Adv., 2012, 2, 8452-8459.

49 C. D. Keenan, M. M. Herling, R. Siegel, N. Petzold, C. R. Bowers, E. A. Rössler, J. Breu and J. Senker, Langmuir, 2013, 29, 643-652.

50 V. McGahay and M. Tomozawa, J. Non-Cryst. Solids, 1989, 109, 27-34.

51 J. H. Markis, K. Clemens and M. Tomozawa, J. Am. Chem. Soc., 1981, 64, C-20.

52 Jmol: an open-source Java viewer for chemical structures in 3D, http://www.jmol.org.

53 V. Marry and P. Turq, J. Phys. Chem. B, 2003, 107, 1832-1839.

54 E. Ferrage, B. Lanson, B. A. Sakharov, N. Geoffroy, E. Jacquot and V. A. Drits, Am. Mineral., 2007, 92, 1731-1743.

55 M. L. Martins, W. P. Gates, L. Michot, E. Ferrage, V. Marry and H. N. Bordallo, Appl. Clay Sci., 2014, 96, 22-35.

56 E. Ferrage, B. Lanson, N. Malikova, A. Plançon, B. A. Sakharov and V. A. Drits, Chem. Mater., 2005, 17, 3499-3512. 